Article

\title{
Hysteresis Analysis and Control of a Metal-Polymer Hybrid Soft Actuator
}

\author{
Manuel Schimmack ${ }^{1}$, Eduardo E. Feistauer ${ }^{2}$, Sergio T. Amancio-Filho ${ }^{2,3}$ and Paolo Mercorelli ${ }^{1, *}$ \\ 1 Institute of Product and Processinnovation, Leuphana University of Lueneburg, Volgershall 1, \\ D-21339 Lueneburg, Germany; schimmack@uni-leuphana.de \\ 2 Institute of Materials Research, Materials Mechanics, Solid State Joining Processes, Centre for Materials \\ and Coastal Research, Helmholtz-Zentrum Geesthacht, Max-Planck-Str. 1, D-21502 Geesthacht, Germany; \\ feistauer@hzg.de (E.E.F.); amancio@hzg.de (S.T.A.-F.) \\ 3 Institute of Polymer Composites, Hamburg University of Technology, Denicke Str. 15, D-21073 Hamburg, \\ Germany; sergio.amancio@tuhh.de \\ * Correspondence: mercorelli@uni-leuphana.de; Tel.: +49-4131-677-5571; Fax: +49-4131-677-5300 \\ Academic Editor: Chunhua Liu \\ Received: 31 October 2016; Accepted: 13 March 2017; Published: 8 April 2017
}

\begin{abstract}
The number of applications of stimulus-responsive polymers is growing at an impressive rate. The motivation of this contribution is to use a commercially available low-budget silver-coated polyamide (PA6) as a thermo-responsive metal-polymer hybrid soft actuator. Polyamide is a hygroscopic polymer; therefore, its mechanical and physical-chemical properties are affected by exposition to humidity or immersion in water. The effect of water absorption content on the PA6 and silver-coated PA6 monofilament properties, such as mass change and resistance, were evaluated. Moreover, the influence of swelling and shrinking effects on the surface morphology, caused by variations of moisture and water immersion, was investigated. Based on these variations, the dynamics of the resistance of the hybrid material were analyzed in the context of the proposed hysteresis model. An identification procedure of the hysteresis is presented along with an approximation of the upper and lower bound based on a constrained least square approach. A switching logic algorithm for this hybrid dynamic system is introduced, which makes it possible to structure the non-linear function in a switching mode. Finally, a non-linear integral sliding manifold is proposed and tested to control the resulting force of the actuator.
\end{abstract}

Keywords: soft actuator; metal-polymer hybrid material; switching system

\section{Introduction}

Stimulus-responsive polymers are relevant for the realization of smart actuators. The term smart, in this context, indicates the actuator ability to both sense and actuate. Polymer fiber-based actuators are also called soft actuators because of their low rigidity. The conductive properties that polymer fibers offer after coating make them suitable for a huge range of applications in biomedical engineering, such as conductors in sensors or bio-sensing textiles for healthcare [1,2]. Polymers that respond to different types of stimuli have become available, such as heat (thermo-responsive materials) [3], stress and pressure (mechano-responsive materials), electric current or voltage (electro-responsive materials) [4], magnetic fields (magneto-responsive materials), changes in $\mathrm{pH}$ or moisture (chemo-responsive materials) and light (photo-responsive materials) [5]. The number of proposed applications is growing at an impressive rate. Recently, applications have been proposed where soft actuators are able to solve even complex problems in strategic sectors, such as bio-inspired robotics. For example, a soft actuator mimicking human esophageal peristalsis for a swallowing robot is presented in [6], where the use of soft material gives an opportunity to imitate the soft-bodied 
esophagus and its biological function. The deformation arising from the non-linear material behavior, the structure and the actuation system causes difficulties in modeling and control. The same type of actuators, pneumatic artificial muscles (PAMs), are used in [7]. Shape-memory alloys (SMA), such as Nitinol in the form of wires, are the most well-known artificial muscles. They undergo a phase change with temperature (or stress) that is responsible for actuation. In reference [8] the electroactive polymer actuators (EAPs) are discussed as an alternative. EAPs, as a class of smart and soft actuators, are ideal candidates for bioinspired mechatronic applications due to their physiological compliance and built-in actuation ability [9]. The complete development and analysis of a soft robotic platform based on antagonistic arrangement of circular and longitudinal muscle groups is presented in [10]. Furthermore, nanotubes drawn from a carbon multi-wall nanotube (MWNT) as artificial muscles are of practical interest, as presented in [11]. The authors showed repeatable contractions of these super-coiled polymer (SCP) actuators constructed from MWNT.

Metal-polymer hybrid fibers are the fundamental core for wearable technology, an integral part of smart textiles and have possible applications in aerospace, sports, military and energy fields [12]. Commercially produced synthetic polymers like polyethylene (PE) and polyamide (PA6.6) fibers are important artificial muscle precursors. They combine the ability of reversible contraction, large volumetric thermal expansion and large anisotropy in thermally-induced dimension changes to provide enhanced muscle stroke [13,14]. High performance robotic muscles from conductive PA6.6 sewing thread and the thermo-mechanical and thermo-electric models of the SCP actuators are presented in [15].

In reference [13], artificial muscles from fishing lines and sewing threads are presented as a possible substitute of commercial NiTi shape-memory metal wires, which are expensive and have strongly pronounced hysteresis. This type of current-voltage hysteresis makes them difficult to control because of the variability of its resistance. In particular, using soft actuators, the kinematic and dynamic model of the actuator is typically affected by uncertainties in the parameters, which are difficult to identify. Therefore, they need to be controlled in a robust way. Moreover, because of the current-voltage hysteresis effect, the resistance is a non-linear function, and the control of the system requires specific and more advanced methods.

Traditionally, quantitative feedback theory (QFT) and $H_{\infty}$ methods are used to achieve robust control of linear systems. Recently, these two methods were also applied in the context of non-linear control through some adaptations. An approach in the context of $H_{\infty}$, along with a linear model adaption around an operating point of a shape memory alloy actuator, is presented in [16]. A dielectric elastomer diaphragm actuator to replicate human pulse signals is controlled with $H_{\infty}$ techniques in [17]. The flexible soft actuator proposed in this work is controlled using a sliding mode control (SMC) approach. The system model is formulated as a switching system. Through the introduced thickness $\delta$ of the proposed sliding surface, the switching nature of this approach is moderated. Thanks to its robust property, the sliding mode approach is one of the principal techniques used today for non-linear systems in the presence of disturbances and uncertainties. During the last few years, considerable efforts have been made in this direction. In [18], a sliding surface is adopted. This technique is based on a combination between a sliding surface and an adaptive pre-action. In [19], the authors describe a comparison of two stator-flux and speed observers for a variable structure in a machine drive. More recent literature considers many related aspects with control, monitoring and disturbances in terms of interference in actuators, which are minimized by using sliding mode control. In particular, in [20] a new monitoring system for removing non-linearities and unmodeled disturbances in piezo electric actuators is proposed. In [21], a high-gain sliding mode observer is designed to reduce the effect of disturbances and uncertainties. The suggested algorithm has the capacity to ensure a vanishing average error in estimation in a finite time, even in the presence of model uncertainties.

The structure of the work is the following. Section 2 shows some morphological features of the soft actuator facilitated by scanning electron microscopy (SEM). The influence of swelling and 
shrinking effects on the surface morphology, caused by variations of moisture and water immersion, is investigated. Based on these variations, the dynamics of the resistance of the silver-coated hybrid material were analyzed in the context of the proposed hysteresis model. The parameter identification and modeling of the soft actuator and its hysteresis are presented in Section 3. The approximation of the hysteresis curve is realized using a constrained least squares method. Based on the results, a switching logic algorithm for the hybrid system is proposed. Section 3.2 proposes a non-linear integral sliding approach. Section 4 provides a concise and precise description of the experimental results, with conclusions resolving the paper. The main goal of the research is to use a commercial low budget silver-coated polyamide (PA6) monofilament as a thermo-responsive metal-polymer hybrid soft actuator, which is stimulated with a defined power.

\section{Experimental Procedure}

\subsection{Physical-Chemical Analysis}

In this work, a thermally-expandable silver-coated polyamide 6 (PA6) monofilament (STATEX $\mathrm{GmbH}$, Bremen, Germany) was selected as a metal-polymer hybrid soft actuator.

\subsubsection{Surface Morphology Analysis}

The monofilament morphology was observed by scanning electron microscopy (SEM). The SEM analyses were carried out with Quanta ${ }^{\mathrm{TM}}$ FEG 650 equipment (FEI Company, Hillsboro, OR, USA) with a voltage of $10 \mathrm{kV}$ and a working distance of approximately $15 \mathrm{~mm}$. Before SEM analysis, the non-conductive PA6 monofilaments were sputtered with gold for $30 \mathrm{~s}$ with a current of $65 \mathrm{~mA}$ using Q150R ES equipment (Quorum Technologies Ltd., Singapore). As a result, a nanometric layer of gold is formed on the surface of the sample, making them conductive. Thus, it facilitates their imaging by SEM and the resolution of small surface features. In the case of silver-coated PA6 monofilament, due to the high conductivity of the silver coating, further gold coating was not required. Nevertheless, a lower voltage was set during SEM measurements in order to prevent charging of defective coating regions, where the PA6 was eventually exposed to the electron beam.

\subsubsection{Water Immersion Test}

Polyamide is a hygroscopic polymer; therefore, its mechanical and physical-chemical properties are affected by exposition to humidity or immersion in water. The effect of water absorption content on the PA6 monofilament properties, such as mass change and resistance, were evaluated by the immersion test. The test was based on the DIN EN ISO 62:2008.8 [22].

Before the water immersion test, the uncoated PA6 monofilament and the silver-coated monofilament were heated in an oven at $50.0 \pm 2.0^{\circ} \mathrm{C}$ for $24 \pm 1 \mathrm{~h}$ to remove remaining absorbed water from the delivered samples. In this manner, both evaluated monofilaments have a water-free reference, where the mechanical and physical-chemical properties are not affected by the moisture content. After the drying process, the monofilaments cool down to the ambient temperature of $23.0 \pm 2.0{ }^{\circ} \mathrm{C}$ with a relative humidity of $50.0 \% \pm 5.0 \%$. The initial mass $m_{1}$ was measured with a Sartorius scale (Model CPA225D-0CE, measurement precision of $10 \mu \mathrm{g}$ ). The next step involves immersing the monofilaments in a beaker filled with $300 \mathrm{~mL}$ distilled $\mathrm{H}_{2} \mathrm{O}$. After each of the immersion cycles, the samples were manually dried in a paper tissue to remove excess water. Following this step, the monofilaments were weighted and returned to the distilled $\mathrm{H}_{2} \mathrm{O}$. After $24 \mathrm{~h}$, the $\mathrm{H}_{2} \mathrm{O}$ concentration change $\left(c_{1}\right)$ in relation to the initial mass $\left(m_{1}\right)$ after one hour was calculated as:

$$
c_{1}=\frac{m_{2}-m_{1}}{m_{1}} \cdot 100 \%
$$


and for the subsequent cycles follows as:

$$
c_{n}=\frac{m_{n}-m_{1}}{m_{1}} \cdot 100 \%
$$

For the water absorption investigation, three replicates of the uncoated PA6 monofilaments with average initial mass of $m=103.98 \mathrm{mg} \pm 0.66 \mathrm{mg}$ and three replicates of the silver-coated monofilaments with average initial mass of $m=102.91 \mathrm{mg} \pm 1.03 \mathrm{mg}$ were used.

\subsubsection{Thermogravimetric Analysis}

Thermogravimetric analysis (TGA) was performed to study the changes in the physical and chemical properties of the monofilaments and to assess the maximum absorbed water content as a function of temperature with a constant heating rate. A TGA instrument, TG 209 F3 Tarsus (Netzsch $\mathrm{GmbH}$, Selb, Germany), was used. After $24 \mathrm{~h}$ of water immersion, samples with masses of 21-26 mg were cut with a scalpel from the uncoated PA6 and from the silver-coated monofilaments. Heating temperature in the interval of $20-800{ }^{\circ} \mathrm{C}$ under a continuous nitrogen flux of $20 \frac{\mathrm{mL}}{\mathrm{min}}$. and a constant heating rate of $20{ }^{\circ} \mathrm{C}$ in. were applied and the change in the mass was continuously recorded.

\subsection{Parameter Identification}

\subsubsection{Resistance Analysis}

An important question to be answered in order to better understand the soft actuator response is related to the correlation between water content and resistance. For this analysis, a monofilament with an initial length of $l=100 \mathrm{~mm}$ was selected and submitted to three different experimental conditions, as shown in Table 1. The resistance was measured with a precision digital multimeter HP 34401A (Agilent Technologies, Santa Clara, CA, USA).

Table 1. Overview of the defined conditions to analyze the correlation between the water content and the resistance.

\begin{tabular}{ll}
\hline State 1 & $\begin{array}{l}\text { indicates the dried monofilaments, which were maintained in an oven at } 50.0 \pm 2.0{ }^{\circ} \mathrm{C} \text { for } \\
24 \pm 1 \mathrm{~h}\end{array}$ \\
\hline State $\mathbf{2}$ & $\begin{array}{l}\text { indicates the "as received" monofilaments with stabilized ambient temperature at } 23.0 \pm 2.0{ }^{\circ} \mathrm{C} \\
\text { and a relative humidity of } 50 \% \pm 5 \%\end{array}$ \\
\hline State 3 & $\begin{array}{l}\text { indicates the monofilaments immersed in a beaker filled with } 300 \mathrm{~mL} \text { distilled } \mathrm{H}_{2} \mathrm{O} 24 \pm 1 \mathrm{~h} \text {, } \\
\text { maximum water absorption as previously described for the water immersion test }\end{array}$ \\
\hline
\end{tabular}

\subsubsection{Hysteresis Analysis of Metal-Polymer Hybrid Material}

A low noise B2961A precision source/measure unit (SMU, Agilent Technologies, Santa Clara, CA, USA) was used for the stimulation and characterization of the metal-polymer hybrid soft actuator. It consists of a DC current source, a DC voltage source and an arbitrary waveform generator, which presents $100 \mathrm{nV} / 10 \mathrm{fA}$ resolution. The SMU allows a voltage-current correct measurement. The low noise measurements are guaranteed by triaxial 4-wire Kelvin probes, which eliminate the measurement error caused by a residual lead resistance. Considering that the electrical power is defined as:

$$
P \equiv \frac{1}{T} \int_{t=0}^{t=T} p \mathrm{~d} t=\frac{1}{T} \int_{t=0}^{t=T} i^{2} R \mathrm{~d} t
$$

where the influence of the previously-analyzed resistor $R$ of the monofilament is linear and the controlled current $i$ is squared. The metal-polymer hybrid monofilament is connected to the triaxial 4 -wire Kelvin probes and stimulated by the current $i(t)$. The soft actuator is loaded with a weight of mass $m=2.13 \mathrm{~g}$ while the stimulation process is carried out. 


\section{Metal-Polymer Hybrid Soft Actuator as a Hybrid Dynamic System}

\subsection{Switching Logic Algorithm}

In this section, a switching logic algorithm for a hybrid dynamic system is proposed. Figure 1 shows the hysteresis curve with the upper bound voltage trajectory $u_{\text {upbo }_{\mathrm{m}}}$ and lower bound voltage trajectory $u_{\text {lobo }_{\mathrm{m}}}$ of the soft actuator in combination with the limitation of the input current $i_{\max }$ and the maximum input voltage $u_{\max }$ to protect the soft actuator.

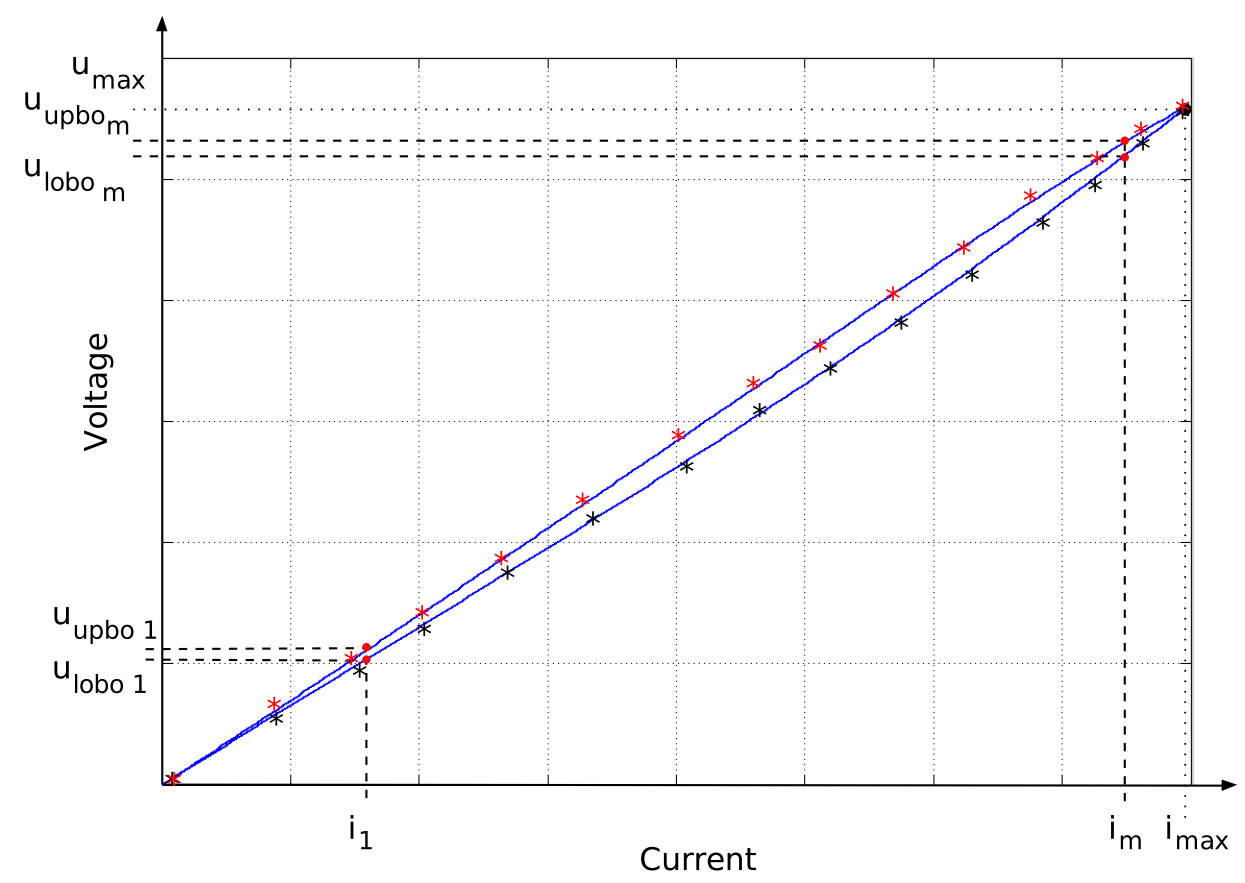

Figure 1. Hysteresis curve after fitting of the upper and lower bound of the metal-polymer hybrid soft actuator with the maximum input voltage $u_{\max }$ and the maximum input current $i_{\max }$.

To find an approximate hysteresis curve using measured data, a fifth order polynomial is used. It represents, in the given interval, the upper and lower bound of input current $i(t)$ and the system voltage $u(t)$. Thus:

$$
u(t)=a_{6}+a_{5} i(t)+a_{4} i^{2}(t)+a_{3} i^{3}(t)+a_{2} i^{4}(t)+a_{1} i^{5}(t)
$$

where $a_{6}=0$ guarantees $u(t)=0$ if $i(t)=0$. The following constraint guarantees that $i_{\max }$ and $u_{\max }$ meet the approximating curve, which is depicted in Figure 1. The first coefficient of the polynomial holds:

$$
a_{1}=\frac{1}{i_{\max }^{5}}\left(u_{\max }-a_{4} i_{\max }^{2}-a_{3} i_{\max }^{3}-a_{2} i_{\max }^{4}-a_{5} i_{\max }\right)
$$

Inserting the coefficient $a_{1}$ in (4), it follows for the voltage that:

$$
u(t)=a_{5} i(t)+a_{4} i^{2}+a_{3} i^{3}(t)+a_{2} i^{4}(t)+\frac{i^{5}(t)}{i_{\max }^{5}}\left(u_{\max }-a_{4} i_{\max }^{2}-a_{3} i_{\max }^{3}-a_{2} i_{\max }^{4}-a_{5} i_{\max }\right)
$$


and:

$$
\begin{aligned}
u(t)= & a_{5}\left(1-\frac{i^{4}(t)}{i_{\max }^{4}}\right) i(t)+a_{4}\left(1-\frac{i^{3}(t)}{i_{\max }^{3}}\right) i^{2}(t)+a_{3}\left(1-\frac{i^{2}(t)}{i_{\max }^{2}}\right) i^{3}(t)+a_{2}\left(1-\frac{i(t)}{i_{\max }}\right) i^{4}(t) \\
& +\frac{u_{\max } i^{5}(t)}{i_{\max }^{5}}
\end{aligned}
$$

Considering the already discussed constrained least squares method in the frame of $m$ measurement data, the following matrix structure is derived in the general way for the lower and for the upper bound:

$$
\left[\begin{array}{c}
u_{1}-\frac{u_{\max } i_{1}^{5}}{i_{\max }^{5}} \\
u_{2}-\frac{u_{\max } i_{2}^{5}}{i_{\max }^{5}} \\
\vdots \\
u_{m}-\frac{u_{\max } i_{m}^{5}}{i_{\max }^{5}}
\end{array}\right]=\left[\begin{array}{cccc}
\left(1-\frac{i_{1}}{i_{\max }}\right) i_{1}^{4} & \left(1-\frac{i_{1}^{2}}{i_{\max }^{2}}\right) i_{1}^{3} & \left(1-\frac{i_{1}^{3}}{i_{\max }^{3}}\right) i_{1}^{2} & \left(1-\frac{i_{1}^{4}}{i_{\max }^{4}}\right) i_{1} \\
\left(1-\frac{i_{2}}{i_{\max }}\right) i_{2}^{4} & \left(1-\frac{i_{2}^{2}}{i_{\max }^{2}}\right) i_{2}^{3} & \left(1-\frac{i_{2}^{3}}{i_{\max }^{2}}\right) i_{2}^{2} & \left(1-\frac{i_{2}^{4}}{i_{\max }^{4}}\right) i_{2} \\
\vdots & \vdots & \vdots & \vdots \\
\left(1-\frac{i_{m}}{i_{\max }}\right) i_{m}^{4} & \left(1-\frac{i_{m}^{2}}{i_{\max }^{2}}\right) i_{m}^{3} & \left(1-\frac{i_{m}^{3}}{i_{\max }^{3}}\right) i_{m}^{2} & \left(1-\frac{i_{m}^{4}}{i_{\max }^{4}}\right) i_{m}
\end{array}\right]\left[\begin{array}{c}
a_{2} \\
a_{3} \\
a_{4} \\
a_{5}
\end{array}\right]
$$

The main idea for the implementation of the switching logic algorithm is to prevent the system from going into saturation. Figure 2 shows the general block diagram of the switching logic algorithm for hybrid systems and its conditions for the lower and upper bound.

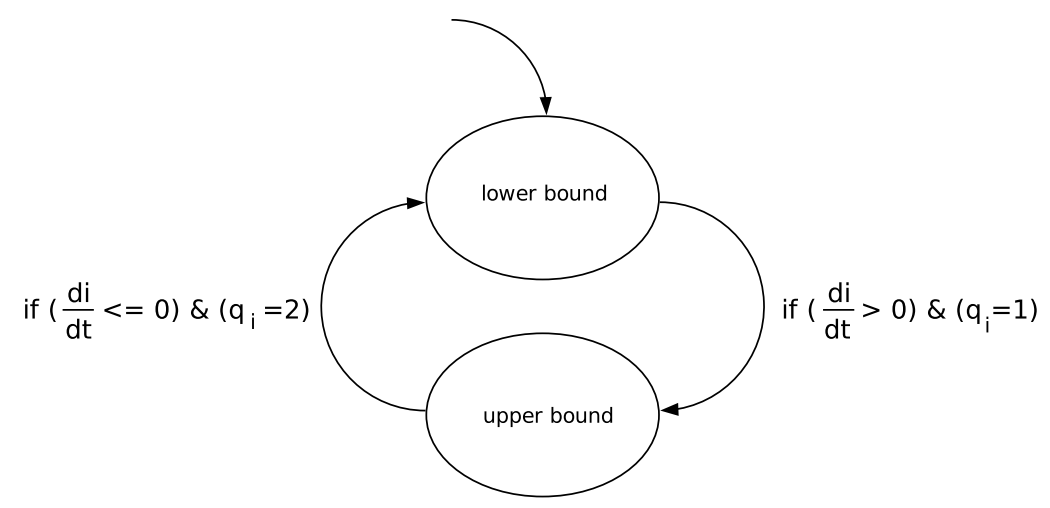

Figure 2. General block diagram of the switching logic algorithm for hybrid systems and its conditions.

\subsection{A Constructive Sufficient Condition for Stability}

Consider the current dynamic of the system as follows:

$$
\frac{\mathrm{d} i(t)}{\mathrm{d} t}=\frac{-f_{i}\left(i(t), \frac{\mathrm{d} i(t)}{\mathrm{d} t}, t\right)+u(t)+\mathrm{d}(t)}{L}
$$

and the resulting force dynamic of the system as:

$$
F(t)=f_{f}\left(i(t), \frac{\mathrm{d} i(t)}{\mathrm{d} t}, t\right)
$$

where $i(t)$ represents the current as the state of the system, the voltage $u(t)$ is a controlled input and $d(t)$ a bounded external voltage disturbance $|d(t)|<D$ with $D \in \mathbb{R}$. 
The model consist of two cascade hysteresis $f_{i}$ and $f_{f}$ of (9) and (10). The two algebraic non-linear functions are structured in a switching mode, with a current-voltage switching parameter $q_{i}$ as follows:

$$
f_{i}\left(i(t), \frac{\mathrm{d} i(t)}{\mathrm{d} t}, t\right)= \begin{cases}f_{i_{\text {upper }}}\left(i(t), \frac{\mathrm{d} i(t)}{\mathrm{d} t}, t\right) & \text { if }\left(\frac{\mathrm{d} i}{\mathrm{~d} t}>0\right) \&\left(q_{i}=1\right) \\ f_{i_{\text {lower }}}\left(i(t), \frac{\mathrm{d} i(t)}{\mathrm{d} t}, t\right) & \text { if }\left(\frac{\mathrm{d} i}{\mathrm{~d} t}<=0\right) \&\left(q_{i}=2\right)\end{cases}
$$

and:

$$
f_{f}\left(i(t), \frac{\mathrm{d} i(t)}{\mathrm{d} t}, t\right)=\left\{\begin{array}{lll}
f_{f_{\text {upper }}}\left(i(t), \frac{\mathrm{d} i(t)}{\mathrm{d} t}\right) & \text { if } & q_{i}=1 \\
f_{f_{\text {lower }}}\left(i(t), \frac{\mathrm{d} i(t)}{\mathrm{d} t}\right) & \text { if } & q_{i}=2
\end{array}\right.
$$

The effect of the current-force hysteresis is directly connected with the current-voltage hysteresis. With this approach, a non-linear integral sliding control is proposed to compensate this effect using a double integral surface:

$$
S(t)=k_{1} e(t)+k_{2} \int_{0}^{t} e(\tau) \mathrm{d} \tau+k_{0} \int_{0}^{t}\left(\int_{0}^{\tau} e(\eta) \mathrm{d} \eta\right) \mathrm{d} \tau
$$

where $e(t)=i_{\mathrm{d}}(t)-i(t)$ with the desired current $i_{\mathrm{d}}(t)$ and the obtained current $i(t)$. The constants $k_{0}, k_{1}$ and $k_{2}$ are positive.

Definition 1. Define the following control law:

$$
u(t)=\hat{u}(t)+k_{3} \cdot \operatorname{sat}\left(\frac{S(t)}{\delta}\right)
$$

where $\hat{u}(t)$ is the sliding equivalent input signal and $\delta$ is a constant that defines the thickness of the boundary layer and with which the chattering phenomenon can be limited. It follows for the saturation function that:

$$
\operatorname{sat}\left(\frac{S(t)}{\delta}\right)=\left\{\begin{array}{l}
1 \quad \text { if } \frac{S(t)}{\delta} \geq 1 \\
\frac{S(t)}{\delta} \quad \text { if }-1<\frac{S(t)}{\delta}<1 \\
-1 \quad \text { if } \frac{S(t)}{\delta} \leq-1
\end{array}\right.
$$

Proposition 1. Consider the system described in (9); when $|S(t)|>\delta$, then the following control law:

$$
u(t)=\hat{u}(t)+k_{3} \cdot \operatorname{sgn}(S(t))
$$

with the sliding equivalent input signal $\hat{u}(t)$ and the sign function $\operatorname{sgn}(\cdot),(16)$ stabilizes the sliding surface defined by (13), if the condition $k_{3}<-|d(t)|$ holds.

Proof 1. Define the following candidate function:

$$
V(t)=\frac{1}{2} S^{2}(t)
$$

considering that:

$$
\dot{S}(t)=k_{1} \cdot \dot{e}(t)+k_{2} \cdot e(t)+k_{0} \int_{0}^{t} e(\tau) \mathrm{d} \tau
$$




$$
\begin{aligned}
\dot{V}(t)= & S(t)\left(k_{1} \cdot \dot{e}(t)+k_{2} \cdot e(t)+k_{0} \int_{0}^{t} e(\tau) d \tau\right) \\
& =S(t)\left(k_{1}\left(\frac{\mathrm{d} i_{\mathrm{d}}(t)}{\mathrm{d} t}-\frac{-f_{i}(\mathbf{x}(t), t)+u(t)+d(t)}{L}\right)+k_{2} \cdot e(t)+k_{0} \int_{0}^{t} e(\tau) \mathrm{d} \tau\right)
\end{aligned}
$$

With (16), the following expression is obtained:

$$
S(t)\left(k_{1}\left(\frac{\mathrm{d} i_{d}(t)}{\mathrm{d} t}-\frac{-f_{i}(\mathbf{x}(t), t)+\hat{u}(t)+k_{3} \cdot \operatorname{sgn}(S(t))+d(t)}{L}\right)+k_{2} \cdot e(t)+k_{0} \int_{0}^{t} e(\tau) \mathrm{d} \tau\right)
$$

Choosing:

$$
\hat{u}(t)=f_{i}(\mathbf{x}(t), t)+L \frac{\mathrm{d} i_{\mathrm{d}}(t)}{\mathrm{d} t}+L \frac{k_{2}}{k_{1}} e(t)+L \frac{k_{0}}{k_{1}} \int_{0}^{t} e(\tau) \mathrm{d} \tau
$$

it follows:

$$
\begin{aligned}
& \dot{V}(t)=S(t) \frac{1}{L}\left(k_{3} \cdot \operatorname{sgn}(S(t))+d(t)\right)=\frac{1}{L}\left(k_{3}|S(t)|+S(t) \cdot d(t)\right)=\frac{1}{L}\left(|S(t)| k_{3}+S(t) \cdot d(t)\right) \\
& \quad \text { If } k_{3}<-|d(t)|, \text { then } \dot{V}(t)<\frac{k_{3}}{L}|S(t)|<0 .
\end{aligned}
$$

\section{Results and Discussion}

In this section, the influence of swelling and shrinking effects on the surface morphology, caused by variations of moisture and water immersion, is investigated. Moreover, based on these variations, the dynamics of the resistance of the hybrid material were analyzed in the context of the proposed hysteresis model.

\subsection{Physical-Chemical Analysis}

\subsubsection{Surface Morphology Analysis}

Figure 3a shows the uncoated PA6 monofilament (State 1, Table 1), and Figure 3b depicts a detailed SEM image of the uncoated fiber surface morphology in the same condition. It is possible to see extrusion defects (marked with white arrows in Figure 3a) and surface ripples (marked with white arrows in Figure 3b). The silver-coated PA6 monofilament is depicted in Figure 4a and the details of its surface in Figure $4 \mathrm{~b}$. Defects of the surface finishing are visible (marked with white arrows in Figure 4a), and a line direction based on the manufacturing process is also seen (marked with red arrows in Figure 4a). Furthermore, in the detailed surface, cracks and rough surface ripples are present (marked with white arrows in Figure 4b). An overview of the morphology of the silver-coated PA6 monofilament in State 1 is depicted in Figure 5a and the details of the silver-coating surface in Figure $5 \mathrm{~b}$ after the stimulation. Defects of the surface finishing are visible (marked with white arrows). It is possible to see, based on the orientation of the marked spots (marked with white arrows), that there is no visible changing of the surface finishing after the stimulation of the fiber. Similar results were obtained for the monofilament stimulated in State 3, as shown in Figure 6. The stimulation process does not change the morphological features of the monofilament, as observed through the comparison of defect orientation and distribution (marked with white arrows) before and after the stimulation (see Figure $6 \mathrm{a}, \mathrm{b}$ in the same sequence). Therefore, it is suggested that defects of the surface finishing are visible (marked with white arrows). It is possible to see, based on the orientation of the marked spots (marked with white arrows), that there is also no visible changing of the surface finishing after the stimulation of the fiber. The dark area in the picture is the distilled water. 

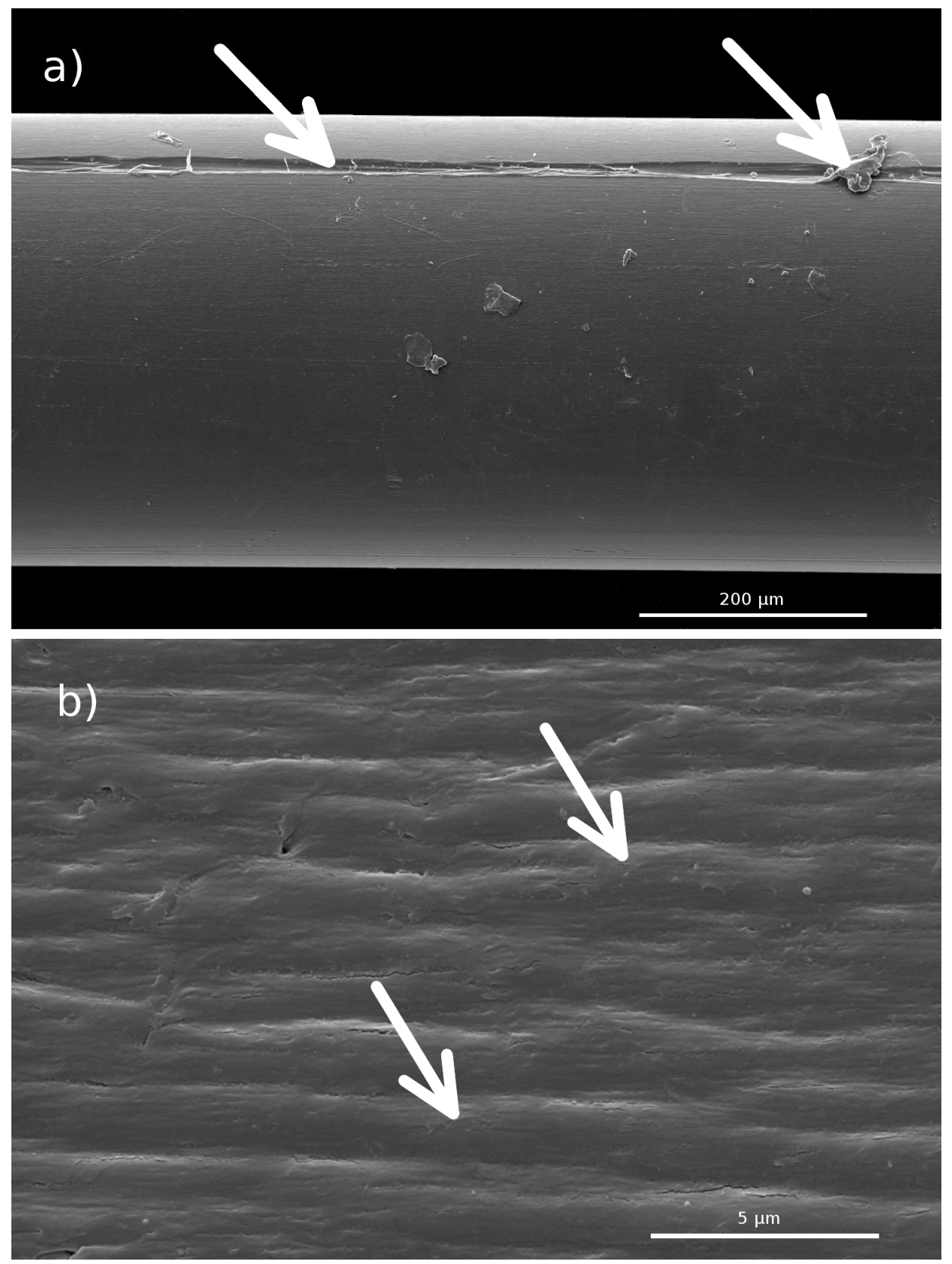

Figure 3. (a) Overview of the morphology of the uncoated PA6 monofilament in State 2 and (b) details of the monofilament surface morphology presented in (a). 

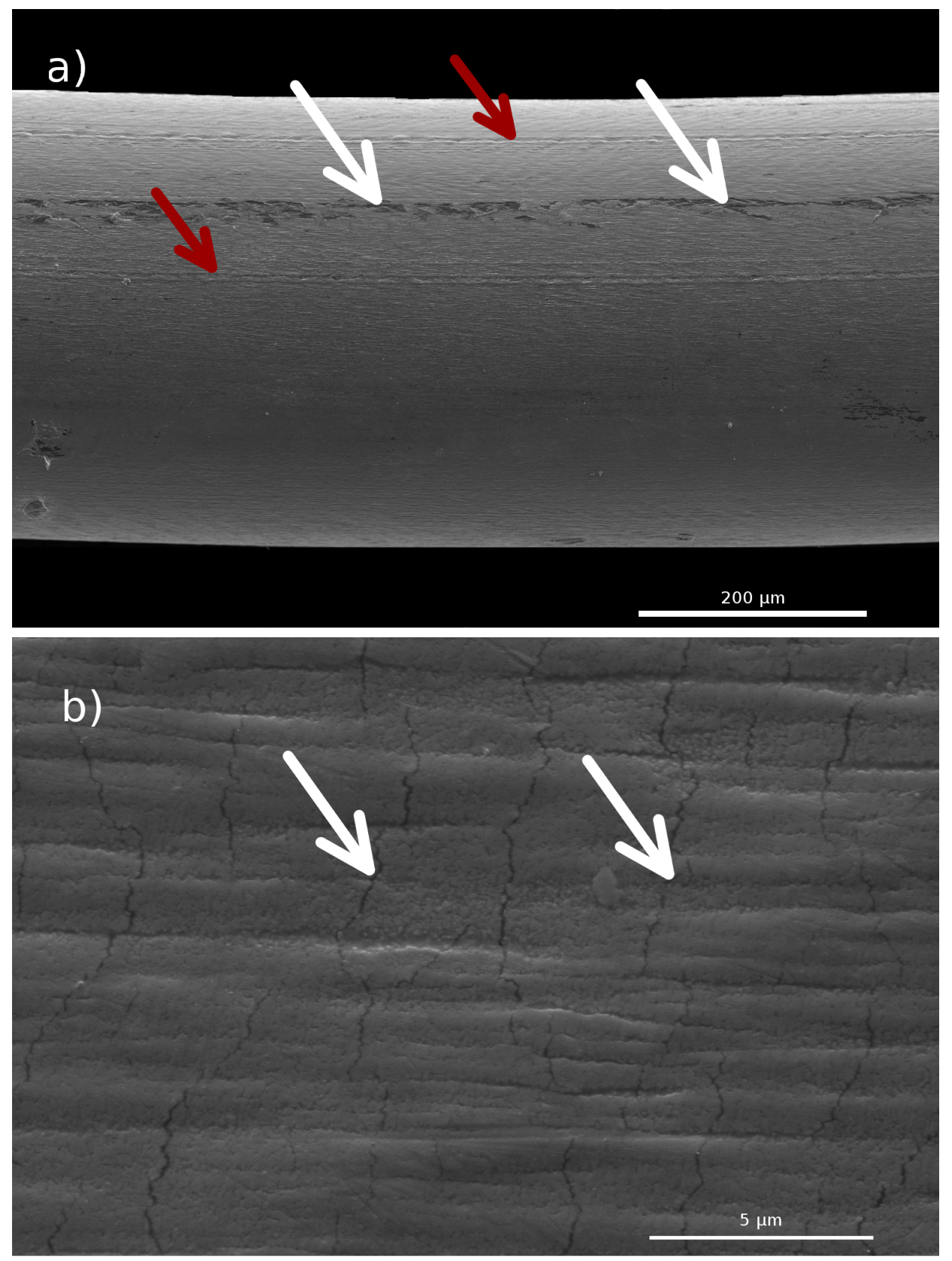

Figure 4. (a) Overview of the morphology of the silver-coated polyamide 6 (PA6) monofilament in State 2 and (b) details of the monofilament surface morphology presented in (a). 

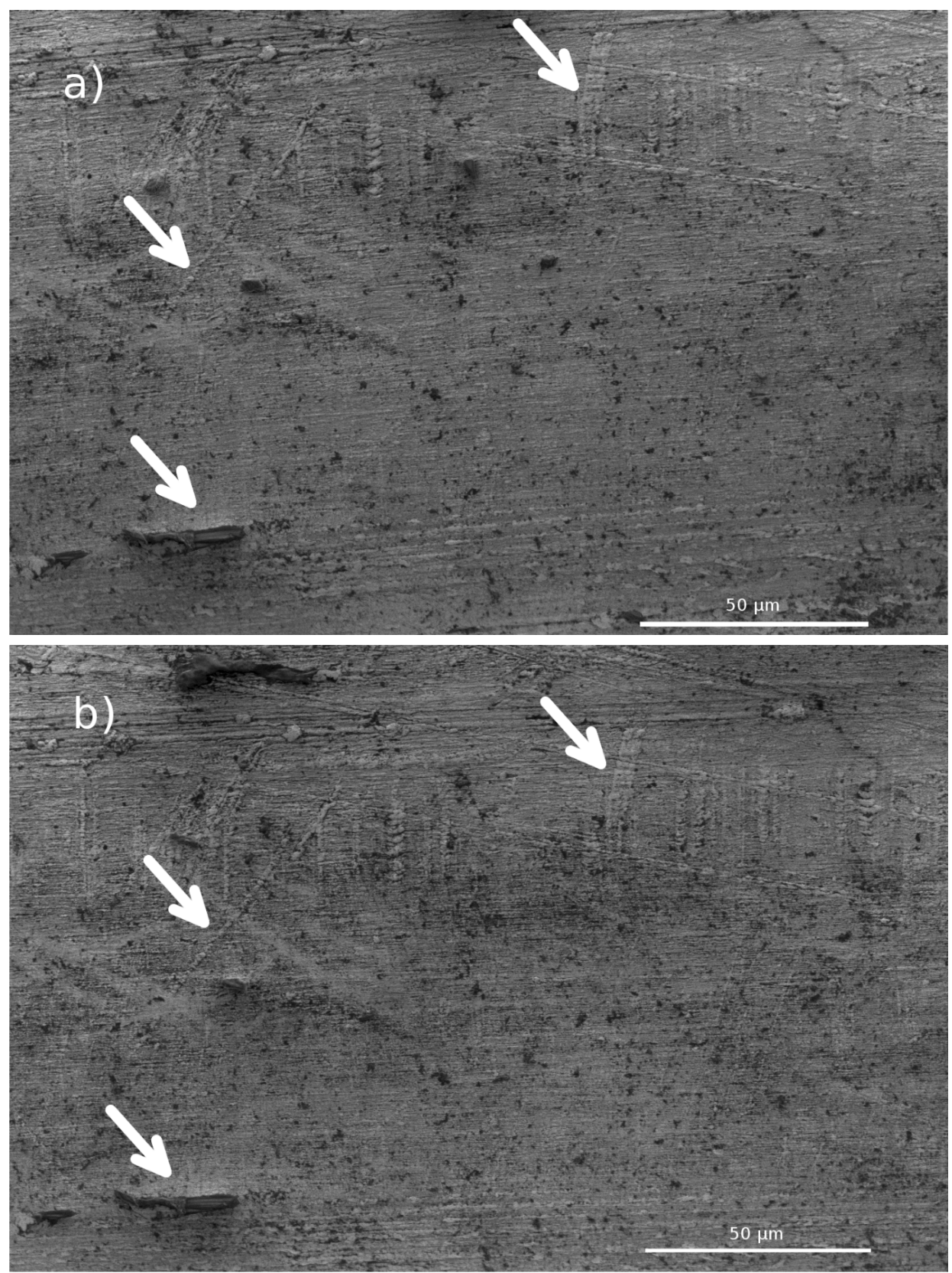

Figure 5. (a) Overview of the morphology of the silver-coated PA6 monofilament in State 1 and (b) details of the monofilament surface morphology presented in (a) after the stimulation. 

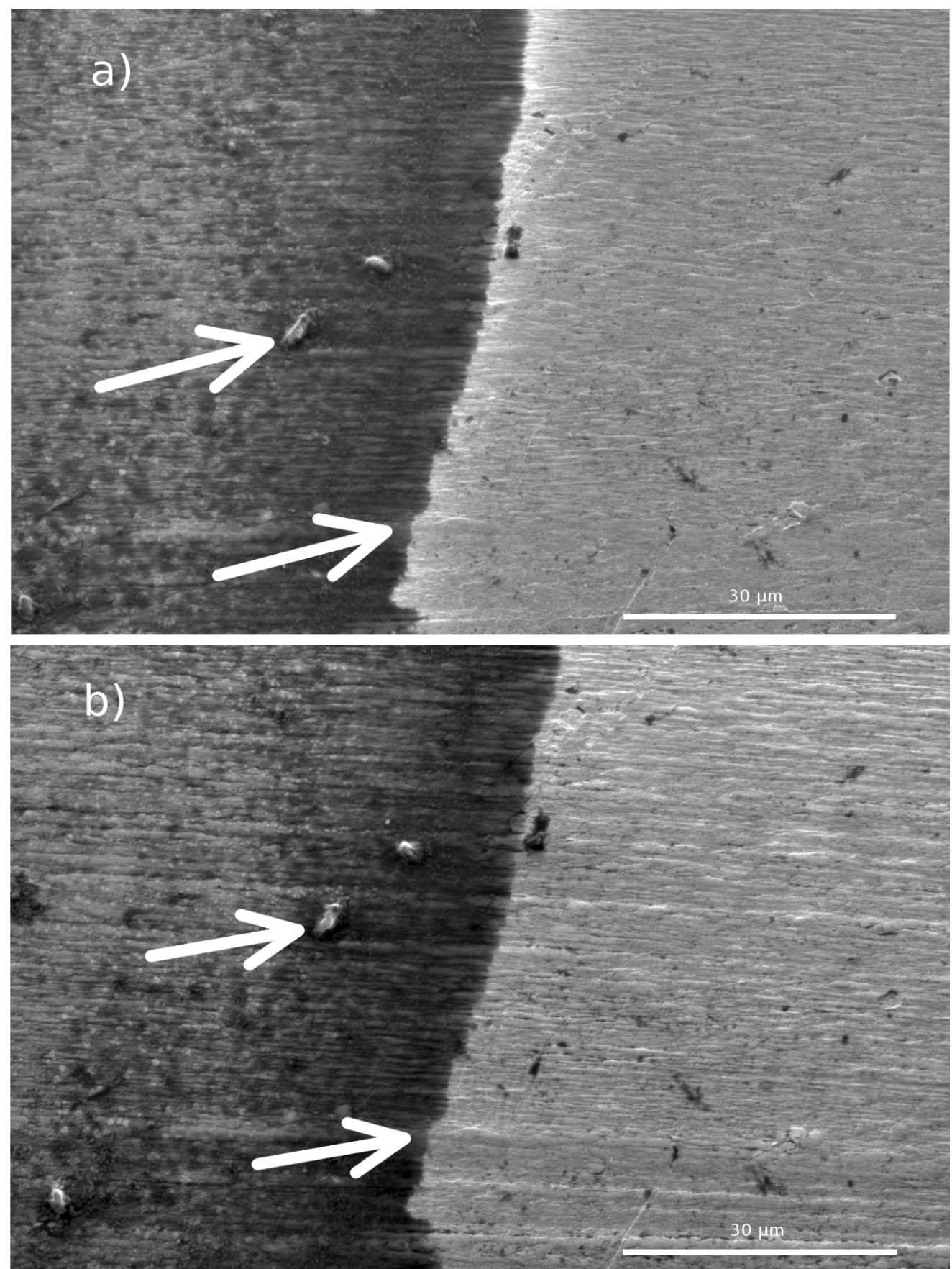

Figure 6. (a) Overview of the morphology of the silver-coated PA6 monofilament in State 3 and (b) details of the monofilament surface morphology presented in (a) after the stimulation.

\subsubsection{Water Immersion Test}

Figure 7 shows a graph of the measured water absorption over time. The water absorption saturation point was observed after $20 \mathrm{~h}$. The uncoated polyamide monofilament absorbed $5.97 \% \pm 0.10 \%$ of water, while the silver-coated monofilament $5.93 \% \pm 0.15 \%$ (measurement at $24 \mathrm{~h}$ ). Therefore, based on the measurements of absorption and its evaluation together with the graphical representations, it can be concluded that the filaments behave very similarly regarding the absorption of water, and the silver-coated monofilament has no effect (or not a detectable one with this experimental setup). 


\subsubsection{Thermogravimetric Analysis}

Figure 8 shows the thermogravimetric curves of the uncoated PA6 and silver-coated PA6 monofilament after $24 \mathrm{~h}$ of water immersion test (State 3). The curve is characterized by the occurrence of two weight loss steps. The first step, between 20 and $200{ }^{\circ} \mathrm{C}$, is formed due to the water evaporation. The measured percent weight losses for both filaments were similar, $\Delta m=6.28 \%$ and $\Delta m=6.34 \%$ for the uncoated PA6 and silver-coated PA6 monofilament, respectively. These values are in agreement with the previous presented water absorption test and correspond to the maximum water content absorbed by the filaments. Moreover, as previously observed, the silver coating does not affect the water absorption mechanism of the PA6 filaments. The second step observed in the TGA thermogram, with the onset at $240{ }^{\circ} \mathrm{C}$, is a result of the final thermo-degradation of PA6, and at this stage, the remaining mass of polymer is lost.

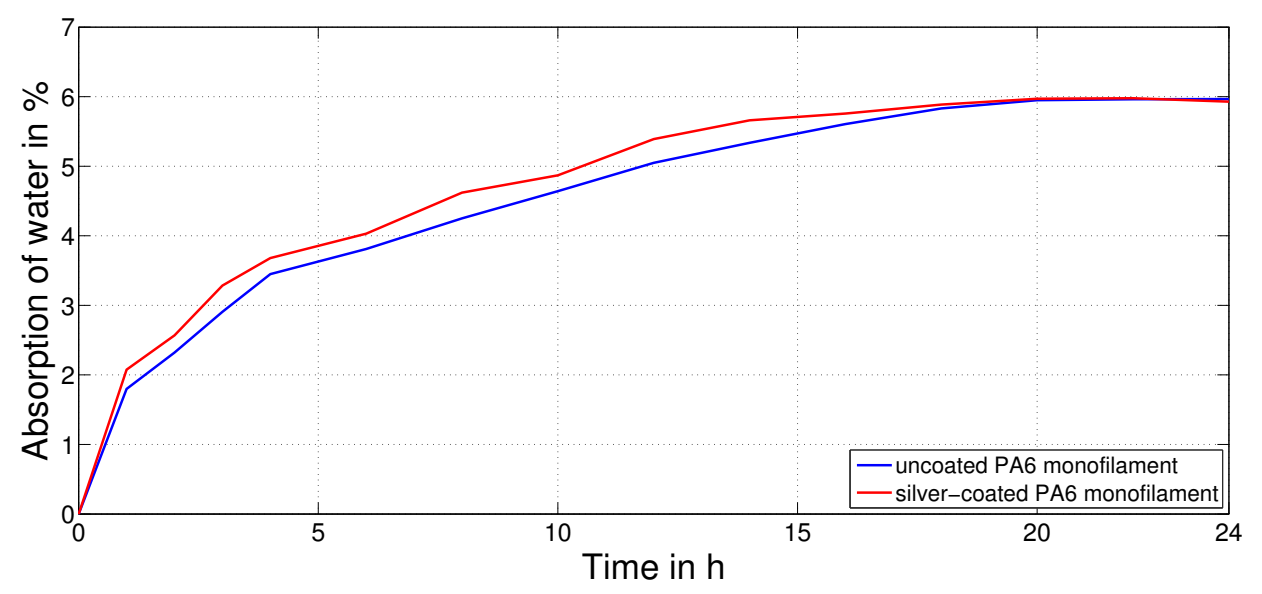

Figure 7. Water concentration after immersion-testing in distilled water of the uncoated PA6 monofilament and the silver-coated PA6 monofilament.

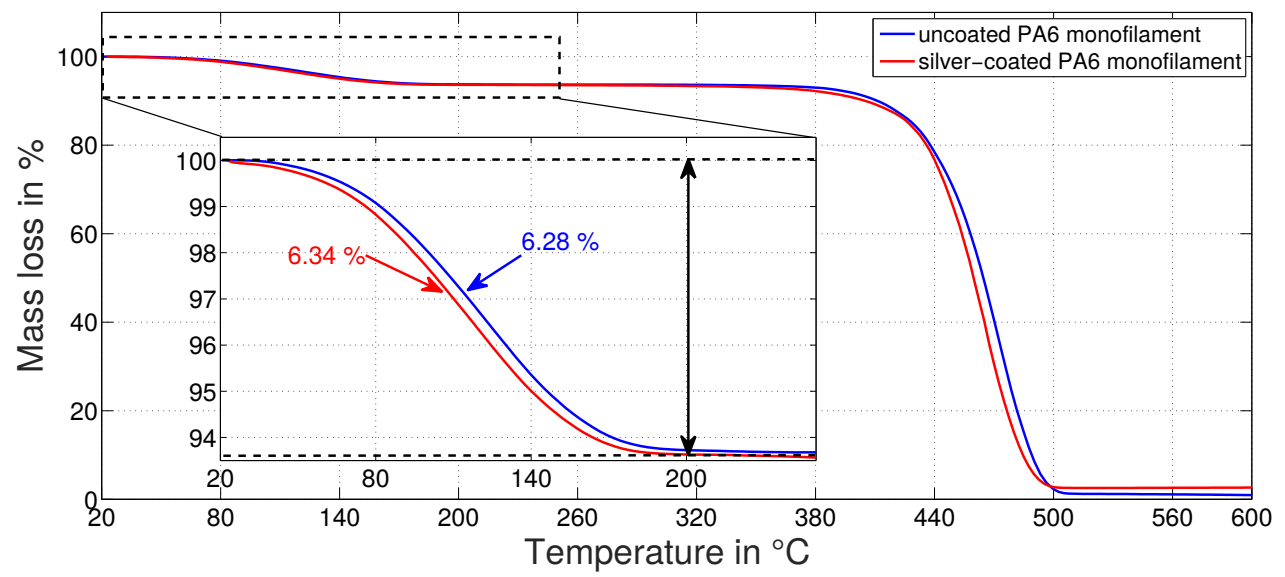

Figure 8. Results of the thermogravimetric analysis (TGA) of the uncoated PA6 in State 3.

\subsection{Parameter Identification}

\subsubsection{Analysis of the Resistance}

Figure 9a shows the measured resistance in the case of States 1,2 and 3 with the standard deviation $\left(\sigma_{x}\right)$ of the metal-polymer hybrid monofilament with a defined length $(l=100 \mathrm{~mm})$. Figure $9 \mathrm{~b}$ shows the results of the weight measurements in the same states. As expected, by drying 
the filaments, a significant decrease in resistance was observed in comparison with States 2 and 3. Therefore, the water content must be taken into consideration when using it as a soft actuator. Ongoing studies are being carried out to better understand this phenomenon. Moreover, one can observe an increase in the mass from State 1 to 3, which can be explained by the water absorbed during the immersion test. Finally, it can be observed that the swelling effect based on the water absorption of the polymer still has no relevant influence on the resistance.
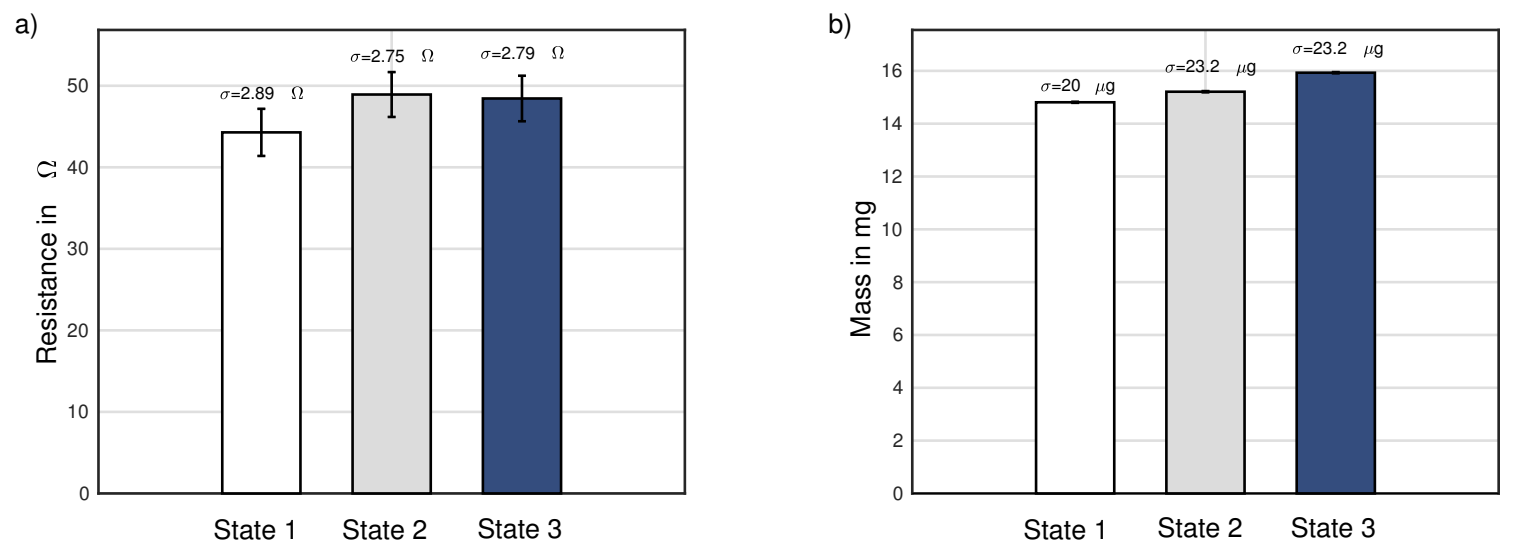

Figure 9. Graphical representation of the measurements of (a) the resistance $R_{n}$ and (b) the mass $m_{n}$ in States 1 to 3 of the soft actuator.

Figure 10a shows the results of the resistance measurements in the case of State 1 after the stimulation process of the samples (which is done by applying an electrical current to heat the metal-polymer hybrid actuator). Figure 10b represents the results of the weight measurements in State 2 and the aforementioned stimulation. It is possible to observe that there is a relevant decrease of about $16.6 \%$ in the resistance for the coated monofilament. However, no variations in mass were observed.

In the previous experimental setup, the effect of the stimulation of State 2 samples was analyzed. The initial state is an intact coating of the monofilament before drying or stimulating. Figure 11a shows the compiled results of the resistance measurements in the mentioned states. The results of the weight measurements under the same states are depicted in Figure 11b. Once more, an accentuated decrease for State 1 samples of $16.4 \%$ in the resistance after stimulation of the coated monofilament (Figure 11a) is detected, while no variation of the mass happened.


Figure 10. Graphical representation of the measurements of (a) the resistance $R_{n}$ and (b) the mass $m_{n}$ in State 2 and after stimulation of the soft actuator. 
a)

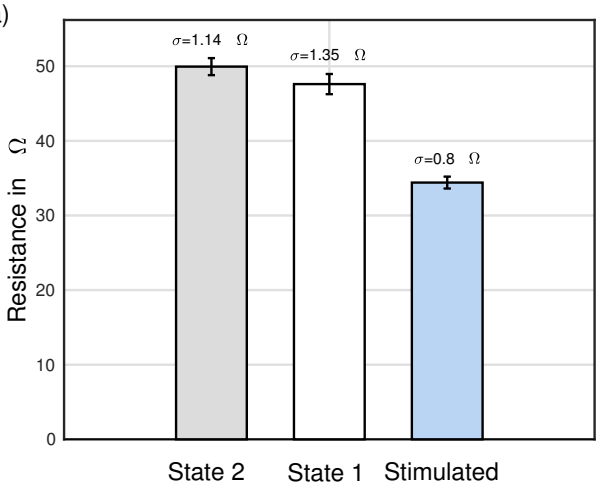

b)

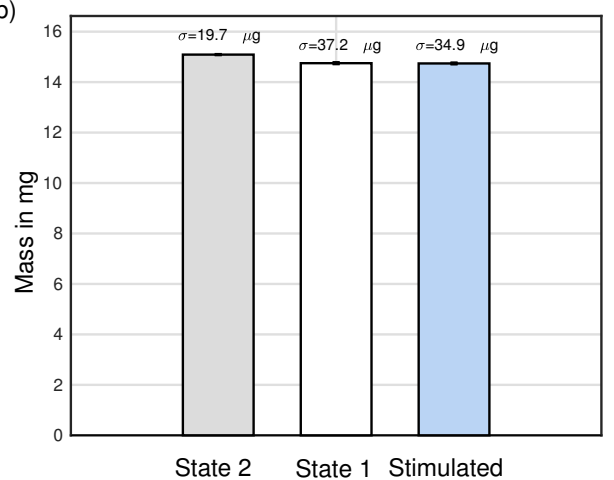

Figure 11. Measurements of (a) the resistance $R_{n}$ and (b) the mass $m_{n}$ in case of the initial States 2 to 1 and after the stimulation process of the replicates.

\subsubsection{Hysteresis Analysis of Metal-Polymer Hybrid Material}

Figure 12a shows the applied input current $i(t)$ as the stimulation signal. The results of the measured power $P(R)$ by the precision SMU are presented in Figure 12b. The metal-polymer hybrid monofilament is connected to the triaxial four-wire Kelvin probes and stimulated by the current $i(t)$. The soft actuator is loaded with a weight of mass $m=2.13 \mathrm{~g}$, while the stimulation process is carried out. Figure 13 shows a considerable variation of the current-voltage hysteresis after the first cycle. This hysteresis curve is stabilized and remains unchanged during the next stimulation cycles. Moreover, the fiber does not return to its original (twisted) configuration.

a)

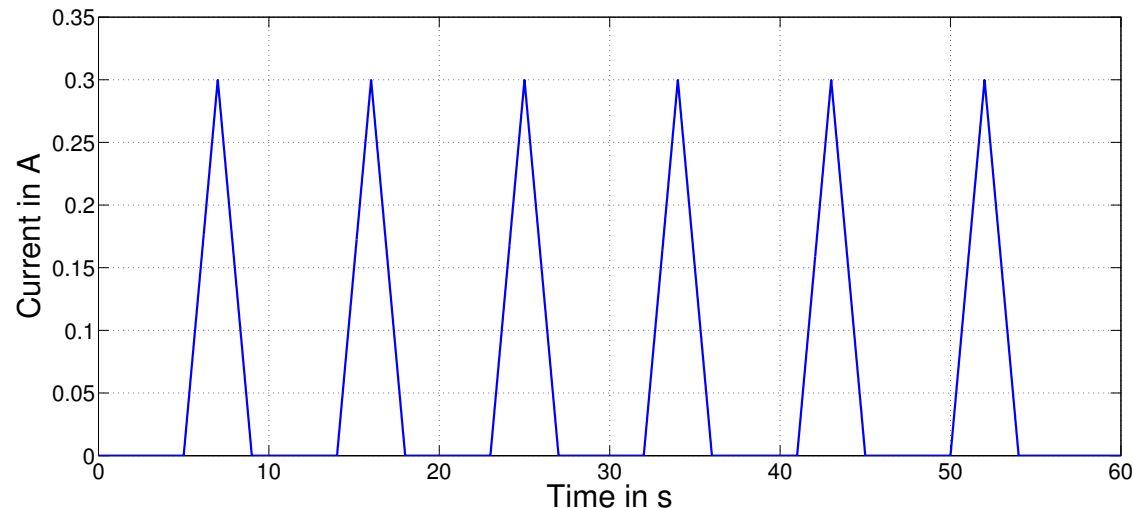

b)

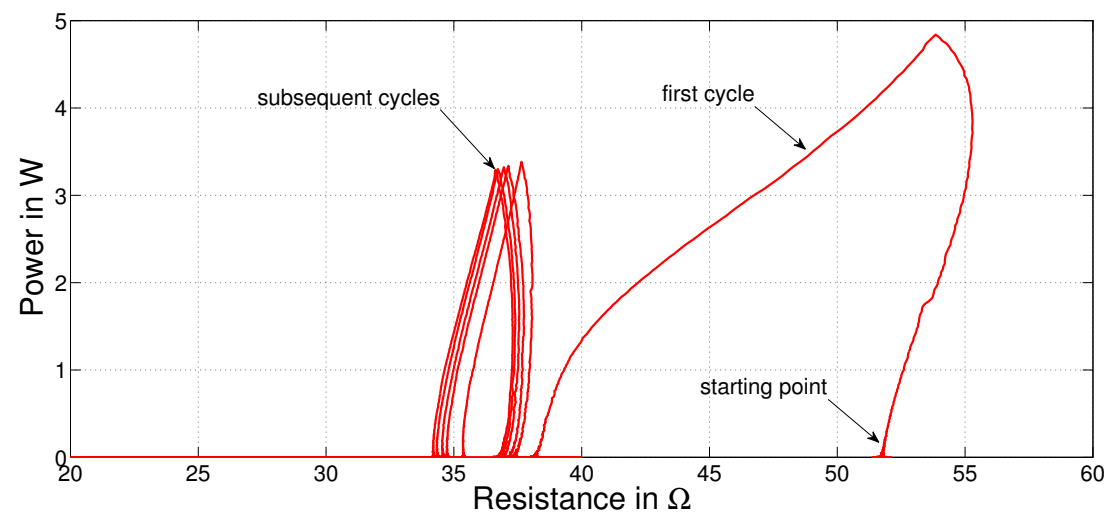

Figure 12. (a) The input current $i(t)$ as stimulation signal and (b) the power $P(R)$ measured by the precision source/measure unit (SMU). 


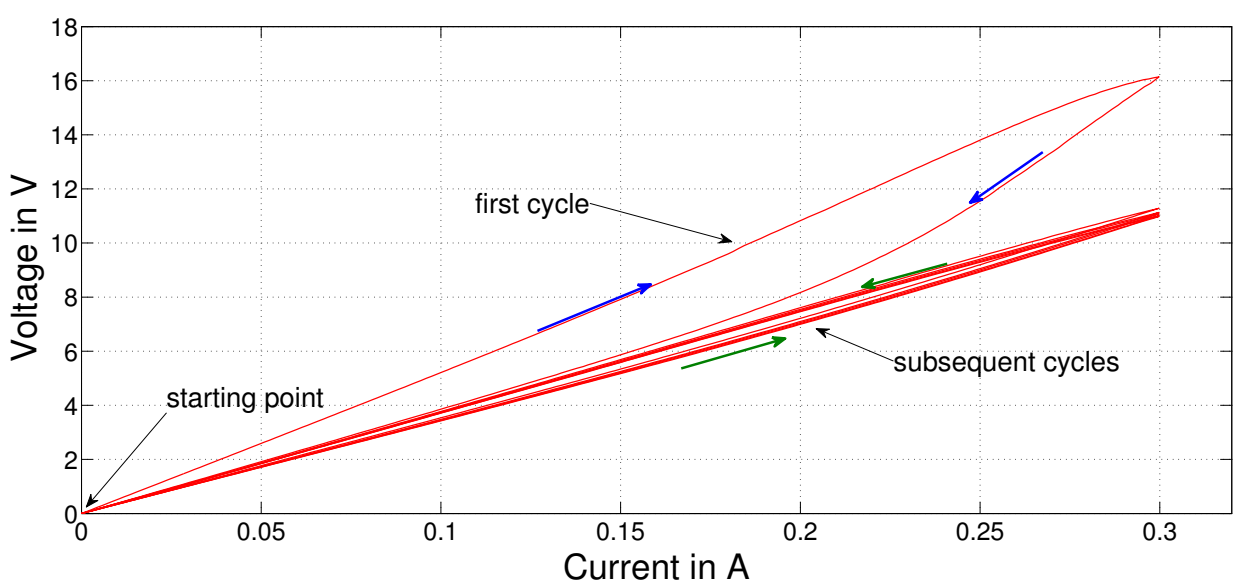

Figure 13. Graphical representation of the measured current-voltage hysteresis of the soft actuator and its upper and lower bounds.

\subsection{Switching Logic Algorithm for Hybrid Dynamic System and a Non-Linear Integral Sliding Mode}

A switching logic algorithm was developed and successfully implemented, based on the previous results of the parameter identification, which makes it possible to structure the non-linear function in switching mode. Finally, the proposed non-linear integral sliding manifold is implemented and tested to control the resulting force of the soft actuator. Figure 14 shows the disturbance $d(t)$ with a peak-to-peak amplitude of $100 \mathrm{mV}$. Figure 15 shows the result of the desired and obtained current in the presence of the disturbance $d(t)$. It is possible to see that the dynamics of the obtained current consist of peaks in the amplitude around $10 \mathrm{~mA}$. This is due to the switching control signal of the sliding approach; see Figure 16. The thickness of the boundary layer of the saturation function was chosen as $(\delta=0.1)$ to guarantee a limit of the chattering phenomenon. In general, thanks to the parameter $\delta$, a compromise between robustness against disturbances (uncertainties) and chattering suppression can be found. The result of the desired and obtained force $f(t)$ of the soft actuator is shown in Figure 17.

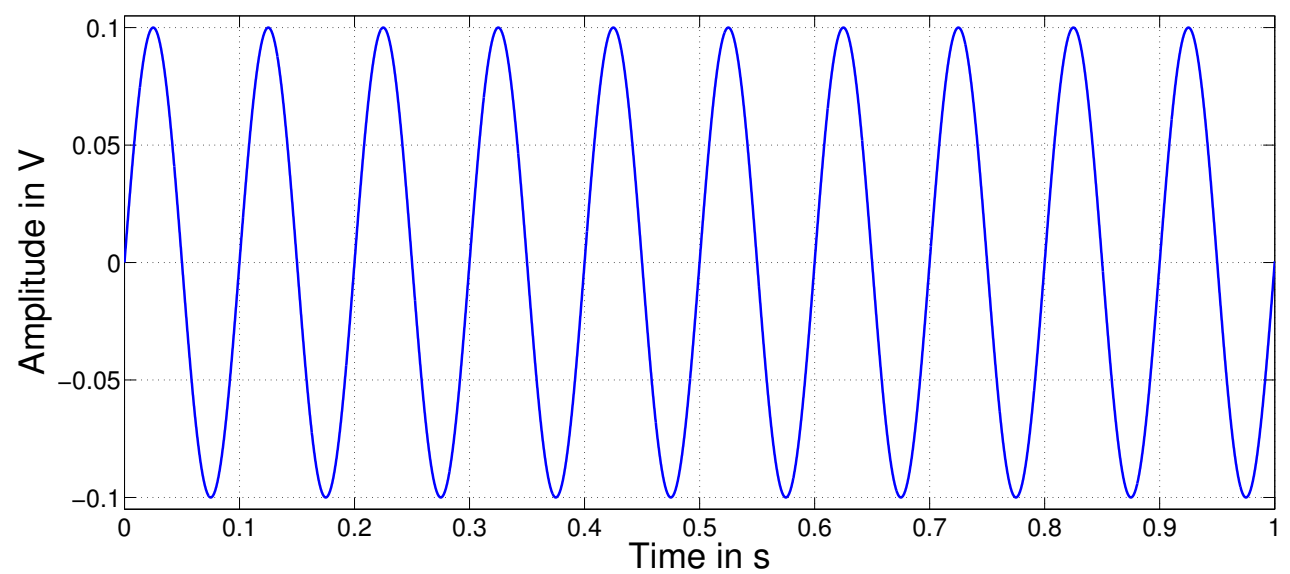

Figure 14. Graphical representation of the voltage disturbance $d(t)$. 


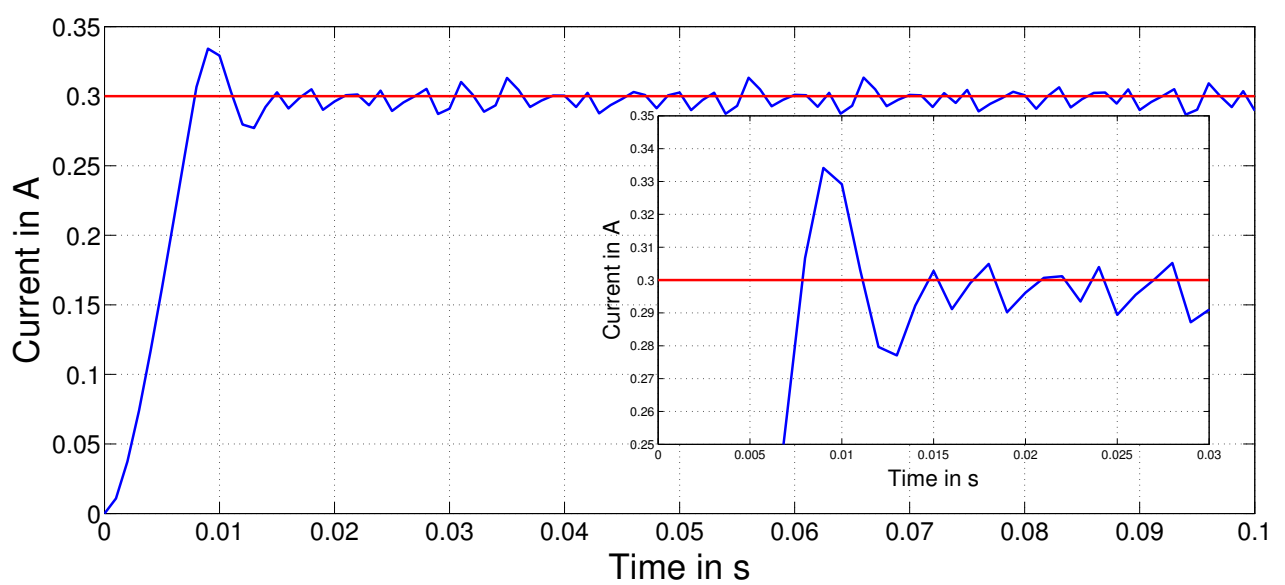

Figure 15. Graphical representation of the desired and obtained current.

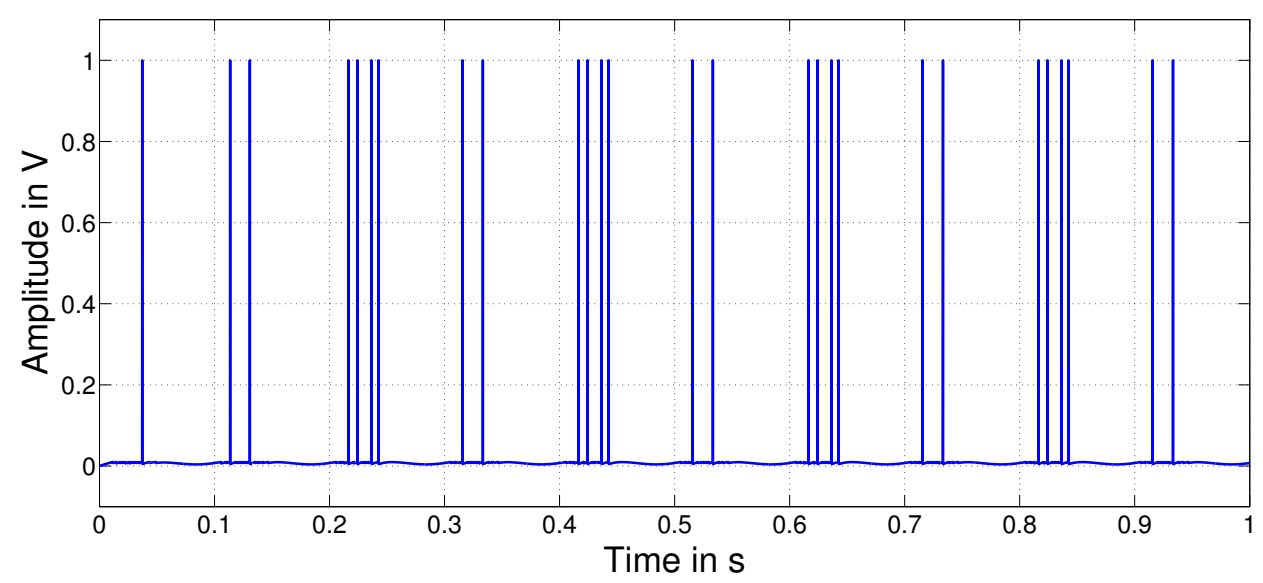

Figure 16. Graphical representation filtered switching variable of the sliding approach.

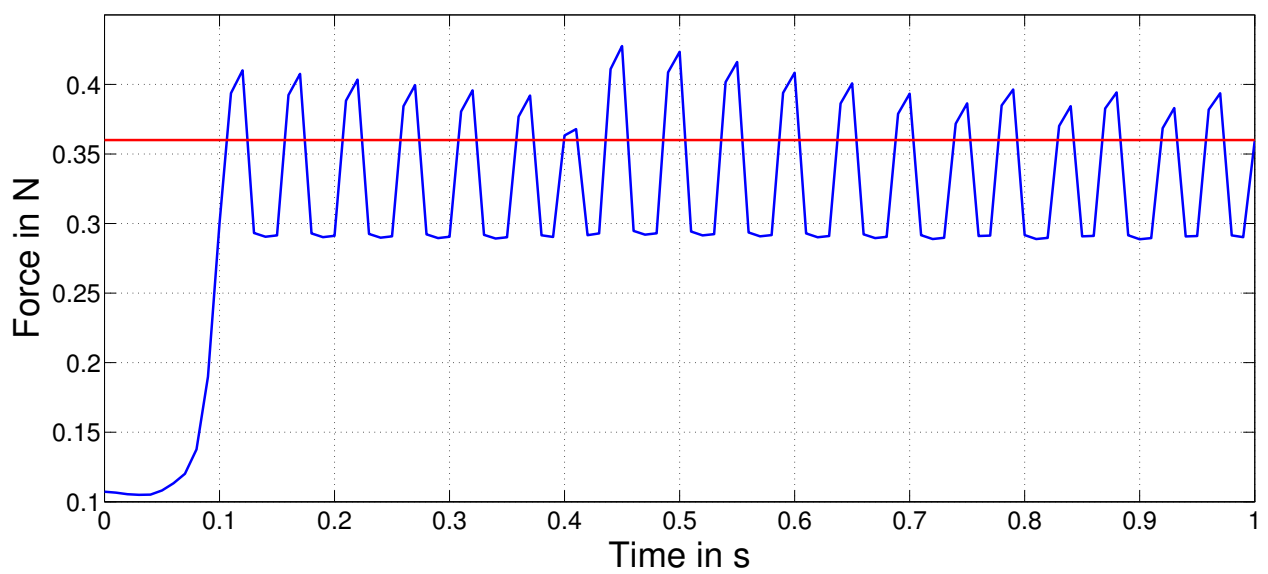

Figure 17. Graphical representation of the controlled force $f$. 


\section{Conclusions}

In this work, a thermally-expandable silver-coated PA6 monofilament was selected as a metal-polymer hybrid soft actuator. The main goal of the research was to use a commercial low budget silver-coated PA6 as a thermo-responsive metal-polymer hybrid soft actuator, which was stimulated with a defined power. Polyamide is a hygroscopic polymer, and the mentioned monofilament was examined under different environmental conditions, more precisely its interaction with water. Through an analysis of the water absorption and of both the coated and uncoated monofilament, it was determined whether or not a coated filament is able to store water. Moreover, the influence of swelling and shrinking effects on the surface morphology, caused by variations of moisture and water immersion, was investigated. Results indicate that the silver coating has no effect (or not a detectable one with the experimental setup) in water desorption.

As expected, the drying process decreased considerably the resistance of the silver-coated filament in comparison with the "as received" (State 2) and immersed in distilled water (State 3). Therefore, the water content must be taken into consideration when applying this PA6 polymer-metal-based soft actuators. Moreover, one can observe an increase in the mass from State 1 to 3, which can be explained by the water absorbed during the immersion test. Finally, it was observed that the swelling effect based on the water content of the polymer still has no relevant influence on the resistance. Based on these variations, the dynamics of the resistance of the hybrid material were analyzed in the context of the proposed hysteresis model.

An identification procedure of the hysteresis was presented along with an approximation of the upper and lower bound based on the constrained least square approach. A switching logic algorithm for this hybrid dynamic system was developed and successfully implemented, which makes it possible to structure the non-linear function in the switching mode. Finally, a non-linear integral sliding manifold was proposed and tested to control the resulting force of the actuator.

Acknowledgments: The authors would like to acknowledge the financial support provided to Eduardo E. Feistauer by the Conselho Nacional de Desenvolvimento Científico e Tecnológico - CNPq (Brazil) and the financial support of the Helmholtz Association through the Young Investigator Group, "Advanced Polymer Metal Hybrid Structures" (Grant no. VH-NG-626). The authors thank Michel L. da Costa for his support in preparing the simulations.

Author Contributions: Paolo Mercorelli conceived the control strategies and the structure of the manuscript; Manuel Schimmack conceived the switching model of the system and the logic algorithm. Together with Paolo Mercorelli he worked on the constructive sufficient condition to obtain the convergence constraints for the control strategy. He designed and performed most of the experiments, analyzed all experimental data and at the end he wrote the manuscript; Eduardo E. Feistauer and Sergio T. Amancio-Filho contributed with polymer-metal fiber characterization (SEM, TGA, water content); All the authors contributed in the preparation, discussions of experimental results and review of the manuscript.

Conflicts of Interest: The authors declare no conflict of interest.

\section{Abbreviations}

SEM Scanning electron microscope

SMU Source/measure unit

TGA Thermogravimetric analysis

EAP Electro-active polymers

PE Polyethylene

SMA Shape-memory alloy 


\section{Nomenclature}

$\begin{array}{ll}a_{m} & \text { Polynomial coefficient } \\ e(t) & \text { Error } \\ d(t) & \text { Voltage disturbance } \\ q_{i} & \text { Switching parameter } \\ k_{0} & \text { Constant } \\ k_{1} & \text { Constant } \\ k_{2} & \text { Constant } \\ k_{3} & \text { Constant } \\ i(t) & \text { Input current } \\ i_{\mathrm{d}}(t) & \text { Desired current } \\ i_{\mathrm{max}} & \text { Limited current } \\ u(t) & \text { Input voltage } \\ \hat{u}(t) & \text { Sliding equivalent input signal } \\ u_{\mathrm{upbo}} & \text { Upper bound voltage trajectory } \\ u_{\mathrm{lobo}} & \text { Lower bound voltage trajectory } \\ R_{n} & \text { Resistance } \\ L & \text { Inductance } \\ S(\mathrm{t}) & \text { Sliding surface } \\ L & \text { Liter } \\ l & \text { Length } \\ m_{x} & \text { Mass } \\ \delta & \text { Thickness of the boundary layer } \\ \mu_{x} & \text { Mean value } \\ \sigma_{x} & \text { Standard deviation }\end{array}$

\section{References}

1. Carpi, F; De Rossi, D. Electroactive polymer-based devices for e-textiles in biomedicine. IEEE Trans. Inf. Technol. Biomed. 2005, 9, 295-318.

2. Schimmack, M.; Hand, A.; Mercorelli, P.; Georgiadis, A. Using a seminorm for wavelet denoising of sEMG signals for monitoring during rehabilitation with embedded orthosis system. In Proceedings of the IEEE MeMeA-International Symposium on Medical Measurements and Applications, Torino, Italy, 7-9 May 2015; pp. 1773-1778.

3. Schimmack, M.; Mercorelli, P. An Extended Kalman Filter as an Observer in a Sliding Mode Controller for a Metal-Polymer Composite Actuator; Springer: Cham, Switzerland, 2005; Volume 427, pp. 305-314.

4. Kruusamäe, K.; Punning, A.; Aabloo, A.; Asaka, K. Self-sensing ionic polymer actuators: A review. Actuators 2015, 4, 17.

5. Hilber, W. Stimulus-active polymer actuators for next-generation microfluidic devices. Appl. Phys. A 2016, 122, 751.

6. Chen, F.J.; Dirven, S.; Xu, W.L.; Li, X.N. Soft actuator mimicking human Esophageal peristalsis for a swallowing robot. IEEE/ASME Trans. Mech. 2014, 19, 1300-1308.

7. Kodama, T.; Okabe, A.; Kogiso, K. Simultaneous estimation of contraction ratio and parameter of McKibben pneumatic artificial muscle model using log-normalized unscented Kalman Filter. In Proceedings of the 2016 IEEE 4th International Conference on Cyber-Physical Systems, Networks, and Applications (CPSNA), Aichi, Japan, 6-7 October 2016; pp. 44-48.

8. Smela, E. Conjugated polymer actuators for biomedical applications. Adv. Mater. 2003, 15, 481-494.

9. Mutlu, R.; Alici, G.; Li, W. A soft mechatronic microstage mechanism based on electroactive polymer actuators. IEEE/ASME Trans. Mech. 2016, 21, 1467-1478.

10. Seok, S.; Onal, C.D.; Cho, K.J.; Wood, R.J.; Rus, D.; Kim, S. Meshworm: A peristaltic soft robot with antagonistic nickel titanium coil actuators. IEEE/ASME Trans. Mech. 2013, 18, 1485-1497.

11. Lima, M.D.; Li, N.; de Andrade, M.J.; Fang, S.; Oh, J.; Spinks, G.M.; Kozlov, M.E.; Haines, C.S.; Suh, D.; Foroughi, J.; et al. Electrically, chemically, and photonically powered torsional and tensile actuation of hybrid carbon nanotube yarn muscles. Science 2012, 338, 928-932.

12. Schimmack, M.; McGaw, D.; Mercorelli, P. Wavelet based fault detection and RLS parameter estimation of conductive fibers with a simultaneous estimation of time-varying disturbance. IFAC Symp. Inf. Control Probl. Manuf. 2015, 48, 1773-1778. 
13. Haines, C.; Lima, M.; Li, N.; Spinks, G.M.; Foroughi, J.; Madden, J.W.; Kim, S.H.; Fang, S.; de Andrade, M.J.; Göktepe, F.; et al. Artificial muscles from fishing line and sewing thread. Science 2014, 343, 868-872.

14. Ramazani, S.; Morshed, M.; Ghane, M. Effect of service temperature on structure and mechanical properties of polyamide 6 \& 66 tyre cords. J. Polym. Res. 2011, 18, 781-792.

15. Yip, M.C.; Niemeyer, G. High-performance robotic muscles from conductive nylon sewing thread. In Proceedings of the 2015 IEEE International Conference on Robotics and Automation (ICRA), Seattle, WA, USA, 26-30 May 2015; pp. 2313-2318.

16. Jayender, J.; Patel, R.V.; Nikumb, S.; Ostojic, M. Modeling and control of shape memory alloy actuators. IEEE Trans. Control Syst. Technol. 2008, 16, 279-287.

17. Ye, Z.; Chen, Z.; Kong, K.W.; Chan, H. Robust control of dielectric elastomer diaphragm actuator for replicating human pulse. In Proceedings of the 2016 IEEE International Conference on Automation Science and Engineering (CASE), Fort Worth, TX, USA, 21-25 August 2016; pp. 188-193.

18. Mercorelli, P. An anti-saturating adaptive pre-action and a slide surface to achieve soft landing control for electromagnetic actuators. IEEE/ASME Trans. Mech. 2012, 17, 76-85.

19. Xu, Z.; Rahman, M.F. Comparison of a sliding observer and a Kalman filter for direct-torque-controlled IPM synchronous motor drives. IEEE Trans. Ind. Electron. 2012, 59, 4179-4188.

20. $\mathrm{Xu}, \mathrm{Q}$. Digital sliding-mode control of piezoelectric micropositioning system based on input-output model. IEEE Trans. Ind. Electron. 2014, 61, 5517-5526.

21. Mercorelli, P. A two-stage sliding-mode high-gain observer to reduce uncertainties and disturbances effects for sensorless control in automotive applications. IEEE Trans. Ind. Electron. 2015, 62, 5929-5940.

22. ISO 62:2008 Plastics-Determination of Water Absorption; International Organization for Standardization (ISO): Geneva, Switzerland, 2008; pp. 1-17.

(C) 2017 by the authors. Licensee MDPI, Basel, Switzerland. This article is an open access article distributed under the terms and conditions of the Creative Commons Attribution (CC BY) license (http:/ / creativecommons.org/licenses/by/4.0/). 